\title{
Search for Leptoquarks at the Tevatron
}

\author{
Pierre-Hugues Beauchemin \\ University of Oxford - Sub-department of Particle Physics \\ Keble Road, Oxford - UK \\ OX1 3RH
}

\begin{abstract}
We present the results for the different leptoquark searches that have been performed at the Tevatron Run II using both the CDF and the D0 detectors [1]. Emphasis will be put on the more recent searches in the $j j \nu \nu$ and $j j \mu \nu$ final states. The latest results for the other channels will be quoted for completeness.
\end{abstract}

\section{Introduction}

The apparent symmetry between the quark and lepton spectra remains unexplained within the Standard Model (SM). However, many theories [2] which go beyond the SM relate these two types of fermions at a more fundamental level. Among them, we can count compositeness models with quark and lepton substructure, grand unified theories (GUTs), based on different gauge groups, and extended technicolour. All these models naturally predict the existence of many different particles which directly couple to a quark and a lepton. These particles are generically referred to as leptoquarks (LQ).

Phenomenological studies of these new particles can be achieved within the framework of effective theories without any knowledge of the more fundamental theories in which they appear. The properties of all possible LQs then follow from the most general lagrangian which describes LQ-fermion interactions $[3,4]$. Such a lagrangian is required to be renormalizable and $S U(3)_{C} \times S U(2)_{L} \times U(1)_{Y}$ gauge invariant. It immediately appears that every LQ carries both a baryon and a lepton number, and belongs to a colour triplet representation of the $S U(3)_{C}$ gauge group. In order to be light enough to be of phenomenological interest at present colliders, LQ couplings have to conserve baryon and lepton numbers separately. This avoids rapid proton decays. Their couplings to fermions also have to be chiral, to preserve the helicity suppression in leptonic pion decays, and family diagonal, to avoid Flavour Changing Neutral Currents. The allowed LQ states can then be classified according to their spin $(S=0,1)$, weak isospin $\left(I_{W}=0, \frac{1}{2}, 1\right)$, electric charge (fractional multiples of $e$ ), fermion number $(F=-2,0)$ and lepton chirality. A total of 24 different LQ states can be possible for each generation [3, 4]. However, only a subset of these possible LQs are generally contained in a particular model. Observation of LQs and determination of their quantum numbers would therefore provide crucial information about the fundamental theory explaining the relation between quarks and leptons.

In $p \bar{p}$ collisions, LQs would be produced in pairs via the strong interaction only. Their production rate is essentially independent of the unknown Yukawa couplings. Scalar LQs therefore depend on one unknown parameter: their mass ${ }^{\mathrm{a}}$. They are further expected to decay into $j j \ell \ell, j j \ell \nu$ or $j j \nu \nu$ final states $(\ell=\mathrm{e}, \mu, \tau)$. Because, at the Tevatron, LQs can be probed at any arbitrary low value of the Yukawa couplings, second and third generation LQs can be produced without having to assume Lepton Flavour Violation, and $q-\nu$ LQs

\footnotetext{
${ }^{a}$ Vector LQs also depend on two anomalous couplings that are related to the magnetic moment and the electric quadrupole moment of the LQs in the colour field [3].
} 
can be observed, searches for LQs at the Tevatron are complementary to those that can be performed at HERA.

In the following, we will summarize the latest results of both CDF and D0 searches for LQs in the different final states. A detailed description of the CDF and D0 detector can be found elsewhere $[5,6]$.

\section{Searches for LQs in the $j j \nu \nu$ final state}

LQs of charge $\pm \frac{1}{3}$ or $\pm \frac{2}{3}$ with left-handed couplings to the lepton can decay to a quark or an anti-quark and a neutrino with a branching fraction of 1 or $\frac{1}{2}$, depending on the type of LQ produced. More than $40 \%$ of all the possible LQs fall into this category. They would all be produced with the same cross section and leave the same signature at the Tevatron: two highly energetic jets and large missing transverse energy (etmiss). Each of the different theories in which LQs arise predict several LQs which share the same dijet+etmiss signature. Hence, this signature offers the potential for the highest yield.

A generic search for an excess of dijet+etmiss events over SM expectations have been performed at $\mathrm{CDF}$ with $2 \mathrm{fb}^{-1}$ of data [7]. Although the measurement constitutes a signaturebased model-independent search, the results have been interpreted in terms of LQs. In order to increase the sensitivity to a wider range of new physics signal, the search has been made independently in two kinematic regimes defined in terms of the etmiss and the scalar sum of the transverse energy of the two jets $\left(H_{T}=E_{T}\left(\right.\right.$ jet $\left._{1}\right)+E_{T}\left(\right.$ jet $\left.\left._{2}\right)\right)$. The choice of the kinematic regions, are displayed in Table 1.

Another key feature of this analysis is that it uses data-driven techniques to estimate the major backgrounds. The dominant sources (more than 90\%) come from $Z \rightarrow \nu \nu+$ jets events and $W \rightarrow \ell \nu+$ jets events where the lepton is lost. $Z \rightarrow \ell \ell+$ jets and $W \rightarrow \ell \nu+$ jets $(\ell=e, \mu)$ events for which the leptons are well reconstructed and for which the + jets requirements correspond to the full set of signal selections are used for the estimate. This significantly reduces the major sources of systematic errors (jet energy scale, PDF, ISR/FSR, underlying events, etc), leaving a statistically dominated uncertainty on the final estimate. The final background predictions for both kinematic regions and the comparisons with the dijet+etmiss observed data are given in Table 1. Observations are consistent with SM expectations, and a 95\% CL upper limit on the $\nu$-j LQ pair production cross section has been computed as a function of their masses. A lower limit on the $\nu$-j LQ masses is obtained by comparing this cross section limit with the NLO theoretical predictions for LQ pair production cross section at the Tevatron [4]. This measurement constrains the LQ masses to be above $177 \mathrm{GeV}$. Note that these limits assume that only one type of LQ contributes to the dijet+etmiss sample. They are therefore conservative.

In the case of a third generation

\begin{tabular}{|c|c|c|c|}
\hline etmiss cut & $H_{T}$ cut & prediction & observation \\
\hline $80 \mathrm{GeV}$ & $125 \mathrm{GeV}$ & $2312 \pm 140$ & 2506 \\
\hline $100 \mathrm{GeV}$ & $225 \mathrm{GeV}$ & $196 \pm 29$ & 186 \\
\hline
\end{tabular}
LQ, where the two jets would come from b-quarks, the signal acceptance is slightly lower than for the first two generation LQs. This results in a limit Table 1: Total number of dijet+etmiss events pre- of $167 \mathrm{GeV}$ on the mass of $\nu$-b LQs. dicted and observed in both kinematic regions.

This is not sufficient to beat the limit of $229 \mathrm{GeV}$ set by $\mathrm{D} 0$ on a dedicated $b \bar{b}+$ etmiss analysis [8]. The fact that this D0 result uses only $425 \mathrm{pb}^{-1}$ of data shows how crucial b-tagging is to such an analysis. 


\section{Searches for LQs in the $j j \mu \nu$ final state}

Among all the 30 possible LQs that could decay into a jet and a neutrino, only four of them could also decay into a muon and a jet (with a branching ratio of $\frac{1}{2}$ ). Searches for LQs in final states consisting of two jets, one muon and large etmiss are therefore sensitive to only those four possible LQs. Although the expected LQ yield is lower than for the dijet+etmiss searches, discovery of LQs in $j j \mu \nu$ events would provide crucial information about the LQ quantum numbers and therefore about the high energy theory which would explain them.

The most recent search for LQs in this

\begin{tabular}{|l|c|c|}
\hline LQ mass & prediction & observation \\
\hline $160 \mathrm{GeV}$ & $6.2 \pm 0.7 \pm 0.8$ & 7 \\
\hline $180 \mathrm{GeV}$ & $6.5 \pm 0.7 \pm 0.8$ & 7 \\
\hline $200 \mathrm{GeV}$ & $6.4 \pm 0.7 \pm 0.8$ & 6 \\
\hline $220 \mathrm{GeV}$ & $6.5 \pm 0.7 \pm 0.8$ & 6 \\
\hline $240 \mathrm{GeV}$ & $6.2 \pm 0.7 \pm 0.8$ & 6 \\
\hline
\end{tabular}
final state has been performed at D0 with $1.0 \mathrm{fb}^{-1}$ of data [9]. This analysis exploits the fact that all the decay products of the produced LQs are visible (the etmiss being attributed to the neutrino) to reconstruct the invariant mass (using the muon and one of the two jets) and the transverse mass (us-

Table 2: Total number of $\mu \nu+$ dijet events predicted and observed for different LQ mass hypothesis. The first error on the prediction is statistical while the second one is systematic. reconstructed invariant mass of the $\mu$-jet system is close (within $100 \mathrm{GeV}$ ) to an assumed LQ mass. Of course, this last selection depends on the LQ mass considered. Although the choice of the cuts has been chosen to optimize the sensitivity to a $200 \mathrm{GeV}$ LQ signal, independent searches have been performed assuming LQ masses between $160 \mathrm{GeV}$ and 240 $\mathrm{GeV}$. The background predictions for the different LQ mass hypothesis considered are given in Table 2. Since these predictions have been obtained with Monte Carlo, the uncertainty is dominated by systematic sources (mostly from the jet energy scale, the $p_{T}$ spectrum of the jets, and the luminosity). The number of observed events for each LQ mass hypothesis tested are also presented in Table 2.

No significant excesses in data over predicted SM background have been observed, and a 95\% CL upper limit on the LQ cross section is estimated as a function of mass. Comparison with NLO cross section calculations allows a lower limit of $214 \mathrm{GeV}$ to be set on the mass of LQs which can couple to both a jet and a muon and to a jet and a neutrino.

Both CDF [10] and D0 [11] have also performed searches for LQs in the dimuon+dijet final state with lower luminosity data samples (less than $300 \mathrm{pb}^{-1}$ ). They set limits of 224 $\mathrm{GeV}$ (CDF) and $247 \mathrm{GeV}$ (D0) on the mass of $\mu$-j LQs. These limits can be combined with those obtained in the $j j \mu \nu$ analysis. The effect of the combination on the $j j \mu \nu$ limits is substantial: the CDF limit increases from $170 \mathrm{GeV}$ to $208 \mathrm{GeV}$, almost reaching the $j j \mu \nu$ D0 limit obtained with 5 times more data. However, the D0 limit on the newest $j j \mu \nu$ LQ search has not yet been combined with the dimuon+dijet results.

\section{Searches for LQs in the other final states}

Besides the LQ searches in the $b \bar{b}+$ etmiss final state that have been presented in section 2 , searches for third generation LQs have also been performed by CDF [12] and D0 [13] with $b \bar{b} \tau \tau$ events. It is interesting to note that in such analyses, the advantage of b-tagging 


\begin{tabular}{|c|c|c|c||c|c|c|}
\hline Final state & $1^{\text {st }}$ gen. & $2^{\text {nd }}$ gen. & $3^{\text {rd }}$ gen. & $1^{\text {st }}$ gen. & $2^{\text {nd }}$ gen. & $3^{\text {rd }}$ gen. \\
\hline$\ell \ell j j$ & $236 \mathrm{GeV}$ & $225 \mathrm{GeV}$ & $151 \mathrm{GeV}$ & $256 \mathrm{GeV}$ & $251 \mathrm{GeV}$ & $180 \mathrm{GeV}$ \\
\hline$\ell \nu j j$ & $205 \mathrm{GeV}$ & $208 \mathrm{GeV}$ & - & $234 \mathrm{GeV}$ & $214 \mathrm{GeV}$ & $-\mathrm{GeV}$ \\
\hline$\nu \nu j j$ & $177 \mathrm{GeV}$ & $177 \mathrm{GeV}$ & $167 \mathrm{GeV}$ & $136 \mathrm{GeV}$ & $136 \mathrm{GeV}$ & $229 \mathrm{GeV}$ \\
\hline
\end{tabular}

Table 3: Summary of the the limits set by CDF and D0 on the LQ masses of each generation, as obtained from searches in different final states containing 0,1 or 2 charged leptons $\ell$.

is more ambiguous than it was for the $b \bar{b}+$ etmiss analyses reported above. In fact, the analysis performed by CDF obtained its best sensitivity to third generation LQs without any identification of the b-jets, while D0 applied some b-tagging. In the end, the importance of b-jets identification is hard to establish as D0 got better limits $(180 \mathrm{GeV})$ than CDF (151 $\mathrm{GeV}$ ), but used three times more data. CDF also performed a similar analysis, but optimized for the case of vector LQs decaying into one tau and one b-jet [14]. As noted in section 2, the limits obtained for such LQs are model dependent as they involve two additional unknown parameters. In the most conservative case, the minimal coupling model, the lower limit on the mass of this type of LQ is $251 \mathrm{GeV}, 100 \mathrm{GeV}$ above the limit obtained for scalar LQs. This difference is due to an enhanced cross section for vector LQs compared to scalar ones.

Table 3 summarizes all the latest limits obtained by CDF and D0 for the different final states in which the different types of LQ could contribute. This table represents all the constraints for each of the three generations of LQs (first generation results are taken from [15]), imposed by the Tevatron on a generic LQ model.

\section{Acknowledgments}

The list of funding institutions which support the CDF and D0 Collaborations can be found in the following references $[5,6]$.

\section{References}

[1] Slides: http://indico. cern.ch/contributionDisplay . py? contribId=96\&sessionId=15\&conf Id=24657

[2] For example: B. Schremp and F. Schremp, Phys. Lett. B 153, 101 (1985); H. Georgi and S.L. Glashow, Phys. Rev. Lett. 32, 438 (1974); E. Eichten and K. Lane, Phys. Lett. B 90, 125 (1980)

[3] J.L. Hewett and T.G. Rizzo, Phys. Rev. D 56, 5709 (1997);

[4] M. Krämer, Talk presented at Beyond the Desert 97, Tegernsee, Germany (1997)

[5] A. Abulencia et al. [CDF Collaboration], J. Phys. G: Nucl. Part. Phys. (2007) 2457-2544

[6] V.M. Abozov et al. [D0 Collaboration], Nucl. Instrum. Meth. A 565463 (2006)

[7] P. Beauchemin, K. Burkett, P. DeVivieiros, E. James, D. MacQueen and P. Savard, CDF note 9170

[8] V.M. Abozov et al. [D0 Collaboration], Phys. Rev. Lett. 99, 061801 (2007)

[9] V.M. Abozov et al. [D0 Collaboration], D0 note 5370

[10] A. Abulencia et al. [CDF Collaboration], Phys. Rev. D 73, 051102 (2006)

[11] V.M. Abozov et al. [D0 Collaboration], Phys. Lett. B 636, 183 (2006)

[12] T. Aaltonen et al. [CDF Collaboration], Fermilab-Pub-08-045-E, Submitted to Phys. Rev. Lett.

[13] V.M. Abozov et al. [D0 Collaboration], D0 note 5447, Submitted to Phys. Rev. Lett.

[14] T. Aaltonen et al. [CDF Collaboration], Phys. Rev. D 77, 092001 (2008)

[15] V.M. Abozov et al. [D0 Collaboration], Phys. Rev. D Rapid Comm. 71, 071104(R) (2005); D. Acosta et al. [CDF Collaboration], Phys. Rev. D 72, 051107 (2005) 